\title{
APPLICATION OF LED ON PLANT GROWTH UNIT IN TOMATO CULTIVATION
}

\author{
Ramazan Şenol, Semra Kiliç ${ }^{1}$ and Kubilay Taşdelen ${ }^{*}$ \\ Isparta University of Applied Sciences, Faculty of Technology, Electrical and Electronic \\ Engineering, Isparta-32200, Turkey
}

Keywords: Chlorophyll, LED plant growth unit, Stomata, Tomato

\begin{abstract}
Effects of Light Emitting Diode (LED) lamps on plant growth with different modulations on the tomato plant were investigated by applying under actual conditions in the greenhouses. Thus, four different pulse width modulation (PWM) systems from red and blue LEDS at different wavelengths were designed. Trials were performed by using direct current (Mode1), $5 \mathrm{Khz}-\varphi=0^{\circ}$ (Mode2), $5 \mathrm{Khz}-\varphi=180^{\circ}$ (Mode3) and $1 \mathrm{Khz}-\varphi$ $=0^{\circ}$ (Mode4) and the tomato grown under the sun at the same conditions were compared for controlling purposes. Stomata movements and total chlorophyll content of the tomatoes were examined, and the results were compared to the tomatoes that were grown under sun. The custom developed artificial four light source system, a treatment of Mode 2 produced the highest yield, while each treatment played its special role in regulating plant morphology and physiology.
\end{abstract}

\section{Introduction}

In winter months tomato cultivation is not suitable due to the insufficient insolation times and low ambient temperatures. Light which was a very important role in the growth and development of the plant, is not enough (Wit and Fankhauser 2016) in these months; an economic loss occurs because tomato production is not performed in the greenhouses. Obtaining crops is not possible especially in the greenhouses where cut flower is grown, in the regions that have less sun light and cold winter months. In this way, the use of different light sources in the greenhouse environment not only helps plant growth, but also allows the plant to use energy efficiently (Zhou et al. 2016). Since the LEDs emit over specific spectral regions, they can be used to regulate the levels of photosynthetically active and photomorphogenic radiation necessary for plant growth and development. The wavelength specificity of LEDs may be used to study the physiological qualities of plants grown in closed plant production systems (Nhut and Nam 2010). ILSys was applied to determine the effect of pulsed light on the fluorescence emission in tomato plants. The fluorescence emission was measured when the plants were radiated with continuous light and light pulsed at different frequencies. The main purpose of a greenhouse was to improve the environmental conditions in which plants are grown. Photosynthesis controlled by complex interactions of physiological, biochemical, and molecular processes can also be determined by anatomical and morphological changes that may be the visible signs of these processes. The benefit of the photosynthesis to the growth of the plant, to obtain the chemical bond energy is very crucial for life events from the energy of light (Taiz and Zeiger 2010). Photosynthesis and transpiration rate can be determined by stomatal responses (Sack and Scoffoni 2013), in which pores play vital roles in the process of exchanging of $\mathrm{CO}_{2}$ and $\mathrm{H}_{2} \mathrm{O}$ (Assmann and Jegla 2016), which are necessary for photosynthesis pathway on leaf surfaces (Esmaeilpour et al. 2016). The present study was undertaken to eliminate such negativities and to provide the light source

*Author for correspondence: <kubilaytasdelen@isparta.edu.tr>. ${ }^{1}$ Suleyman Demirel University, Faculty of Arts \& Sciences, Isparta, Turkey. 
required by the plants. Via these Light Emitting Diode (LED) plant growth units were being developed and tested.

\section{Materials and Methods}

The greenhouse controller block diagram, which is integrated into the system model recommended by Javadikia et al. (2009), is presented in Fig. 1. The total area of the prototype greenhouse was set to 50 sq.m (March 2015 - January 2016). In the set model, input variables were light intensity, temperature, air humidity, soil humidity, wind speed and carbon dioxide level. As for output variables, identification of the light intensity of the LED plant growth unit, irrigation, heating, cooling, and ventilation were handled.

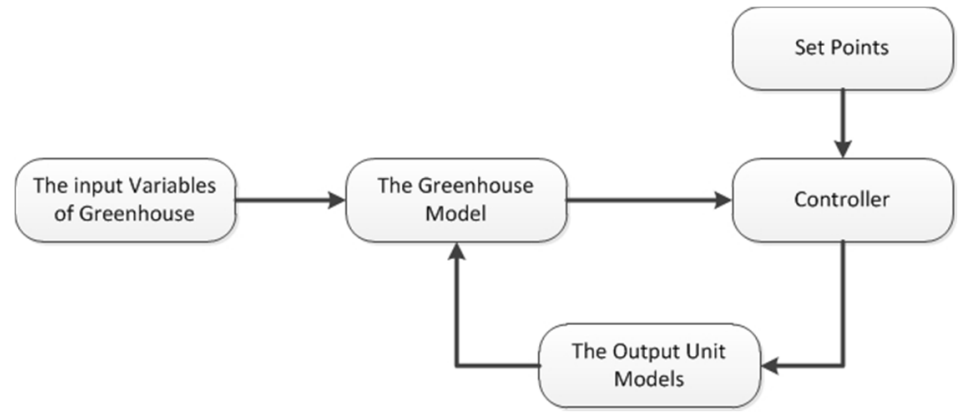

Fig. 1. Greenhouse controller block diagram.

As known from the Fuzzy Logic principles an FLC acts as a non-linear system implementing a human-based reasoning for the computation of their output values (Caponetto et al. 1998). This is mainly due to the flexibility and simplicity by which knowledge can be expressed using fuzzy rules as well as to the theoretical developments in this field (Salgado and Cunha 2005). For all the Fuzzy inferences, Mamdani inference model was preferred as it is both easy and suitable for the greenhouse model. Triangle fuzziness method was chosen.

The communication between the PWM generation card and LED driver card in the LED plant growth unit was provided via an optocoupler. Thus PWM unit and the driver unit were electrically isolated from each other. Four different working modes specified for four LED plant growth units were identical and used in the experiments. One of them is Direct current (Mode1) and it is based on the continuous powering of the LEDS. Second working mode was the PWM (5Khz- $\varphi=0^{\circ}-$ Mode2) mode. In this mode, red and blue leds are powered at the same time for 100 microseconds and $5 \mathrm{kHz}$ frequency was obtained. In the third working mode, red and blue led groups were powered separately with a phase difference of $180^{\circ}$ between them. Application frequency was again $5 \mathrm{kHz}\left(5 \mathrm{Khz}-\varphi=180^{\circ}-\right.$ Mode3). In the final working mode, red and blue LEDS were powdered at the same time for 0,5 milliseconds and $1 \mathrm{kHz}$ frequency was obtained $\left(1 \mathrm{Khz}-\varphi=0^{\circ}\right.$ Mode4). In all the study modes average light intensity reaching to the plant surface occurred as 20 $\mu \mathrm{mol} \mathrm{m} \mathrm{m}^{-2} \mathrm{~s}^{-1}$ (Fig. 2). In the greenhouse, the areas where 4 different light and control applications were made were separated from each other by the sections. The 25-day tomato seedling was planted in equal numbers (50 pieces) each. After the plants have been adjusted for orientation, the application was initiated. At the end of 6 months application, appropriate samples were taken from tomato plants, then stomata and chlorophyll were measured. 


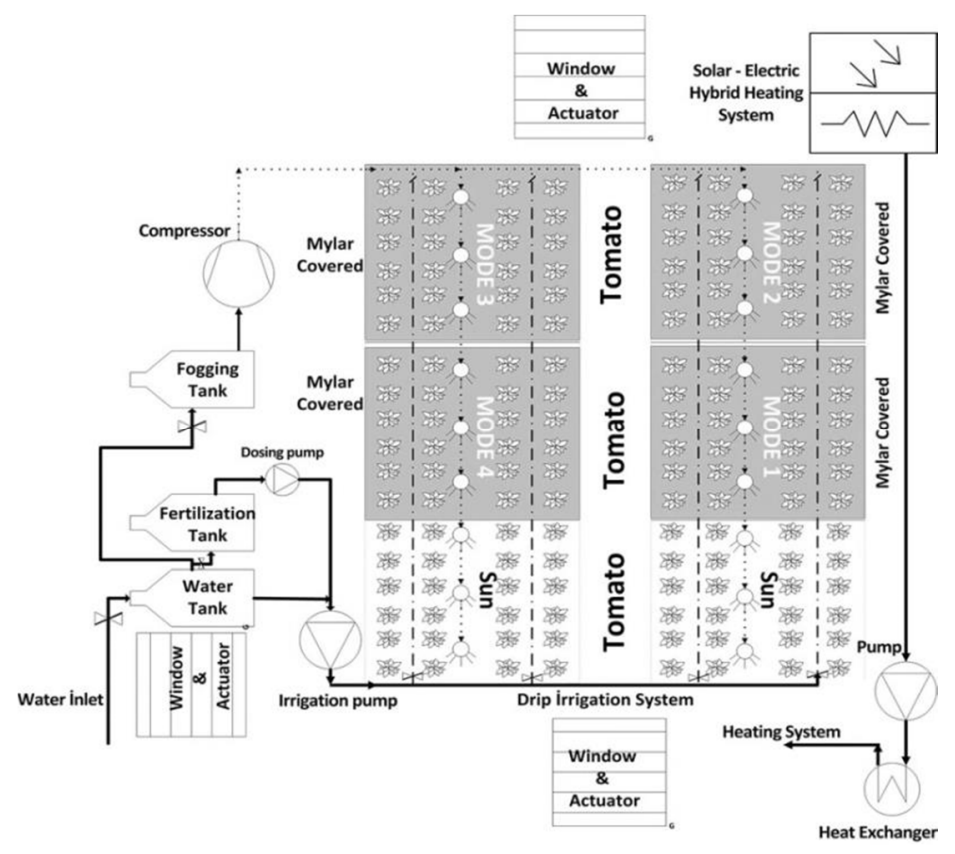

Fig. 2. Mechanization in the greenhouse.

Epidermal tissue was stripped from the adaxial and abaxial surfaces of leaf lamina pieces to determine leaf stomatal density, expressed as the number of stomata per unit leaf area, mounted on a glass slide and immediately covered with a coverslip, and then lightly pressured with finepoint tweezers. For each independent measurement, number of stomata $(s)$ and epidermal cells $(e)$ for each film strip was counted from both the adaxial and abaxial surfaces in a $0.04 \mathrm{~mm}^{2}$ unit area (50 microscopic field). The leaf stomatal index (SI) was calculated according to Rengifo et al. (2002). Stomata magnitudes were defined in $\mu \mathrm{m}$ using an ocular micrometer under light microscope (40 $\mathrm{x}$ 10). Total chlorophyll contents were calculated using a chlorophyll meter (Minolta SPAD-502). The statistical analysis was carried out by one-way classification of ANOVA to describe the growth parameters and to calculate statistical significance.

\section{Results and Discussion}

Effects of different light applications (Mod 1, Mod 2, Mod 3 and Mod 4) on growth and development of tomatoes were determined by measuring the morphological, anatomical and physiological parameters.

At different light levels stomata and epidermis numbers on the adaxial surface of the leaves showed a significant difference when compared to the control. Epidermis and stomata numbers showed14 and 60\% increase in mode1, 38 and $76 \%$ in mode 2, 4 and $46 \%$ in mode 3 . Mode 4 application has lower epidermis and stomata numbers than the control plants $(\mathrm{p}<0.05)$. Also worse data of Mode 4 application than the control showed that this application is not successful. Stomata and epidermis numbers are also examined in the abaxial surface of the leaves and Mode 2 application has given the best results when compared to the control and other applications (Fig. 3). As a result when all the trials were compared to the control group the best result was obtained in Mode 2 application. The differences in the densitiy of stomatal apertures, especially in diverse 
stomata count and sizes of the stomatas are some of the important factors that enable to grow the plants under various conditions. Furthermore, the relationship between the light density and the convertion of the meristemoids into stomatas in both surfaces of the leaf showed the number of stomatas are greater compared to other studies.
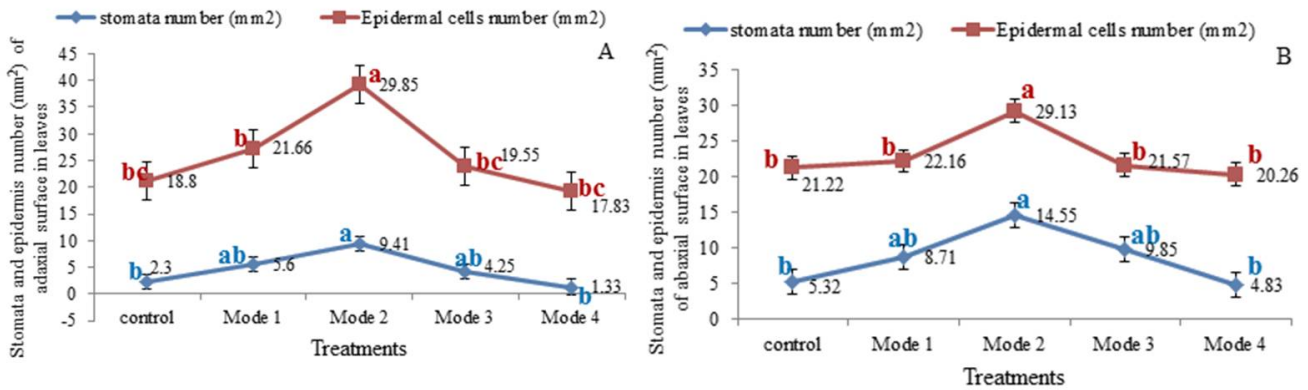

Fig. 3. Stomata and epidermis number $\left(\mathrm{mm}^{2}\right)$ of adaxial (A) and abaxial (B) surfaces of leaves of tomato plants treated with different light.

According to the application results, stomata index was calculated on the adaxial and abaxial surfaces of the leaves and the results were obtained at a relatively significant level when compared to the controls (Fig. 4). Accordingly the best results on both the adaxial and abaxial surfaces of the leaves were obtained in mode 2 application. Moreover this result is specified as $38 \%$ on the adaxial surface of the leaf than the control and $61 \%$ on the abaxial surface of the leaf. Other applications except Mode 4 have given higher results than the control but the increase ratio was not observed as in mode 2 application. In Mode 4 stomata index on both surfaces of the leaves were resulted with lower data. In this case, when the applications were compared to the controls the best results were obtained in Mode 2 application and the best results were obtained in Mode 4 application $(\mathrm{p}<0.05)$. Stomatal control is provided by the regulation of the stomatal apertures and obtaining $\mathrm{CO}_{2}$ (Zhao et al. 2015). The effects of the application factors on the growth of the plant is directly proportional to the stomatal index.

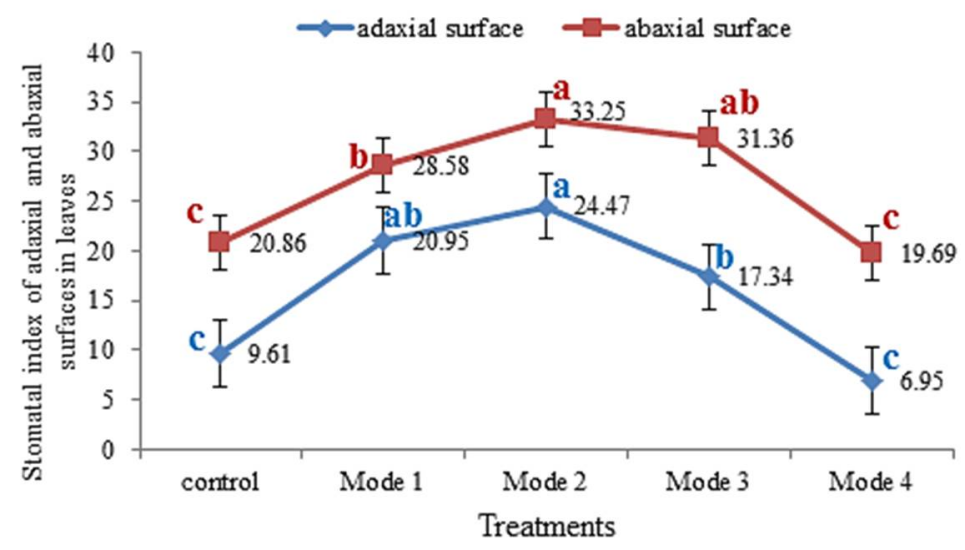

Fig. 4. Stomatal index in adaxial and abaxial epidermis of leaves of tomato plants treated with different light. 
At different light applications sizes of stomata were examined on both surfaces of the tomato leaves and when the results for the adaxial surface of the leaves were compared to the control the largest stomata were found in Mode 2. Thus the stomata length on the adaxial surface of the leaves has shown 11\% increase in Mode 1,33\% in Mode 2 and 2\% in Mode 3. In Mode 4 application, stomata that were smaller than the controls, the observed ratio is specified as a $9 \%$ decrease than the control $(\mathrm{p}<0.05)$. When the stomata width is compared to the control Mode 1, Mode 2 and Mode 3 applications showed an increase by 39, 40 and 11\%); it was determined that stomata size was smaller than the control in Mode 4 application (10\%). It was observed that there was a relatively significant growth on the stomata length on the abaxial surface of the leaves when compared to the control in Mode 2 application and other applications (44\%) (Fig. 5). In the other applications it is specified that the increase occurs at varying ratios. Interestingly the stomata length on the abaxial surface of the leaf was at a higher value than the control $(\mathrm{p}<0.05)$. When the other parameters were examined Mode 4 application has shown lower values than the control, it was found that stomata length on the abaxial surface of the leaf was higher than the control. This ratio is determined by observing $21 \%$ increase in the stomata length in Mode 2 application when compared to the control. When the abaxial stomata width of all the applications were examined, it was specified that they did not have very different values than the control and only Mode 2 application was a bit different from the control. This ratio was determined with $29 \%$ increase. Other applications have almost similar values with the control application. However Mode 4 application, as in other parameters, except the abaxial stomata length of the leaf, is resulted with a lower value than the control application. Thus abaxial stomata width of the leaf was $12 \%$ smaller than the control.
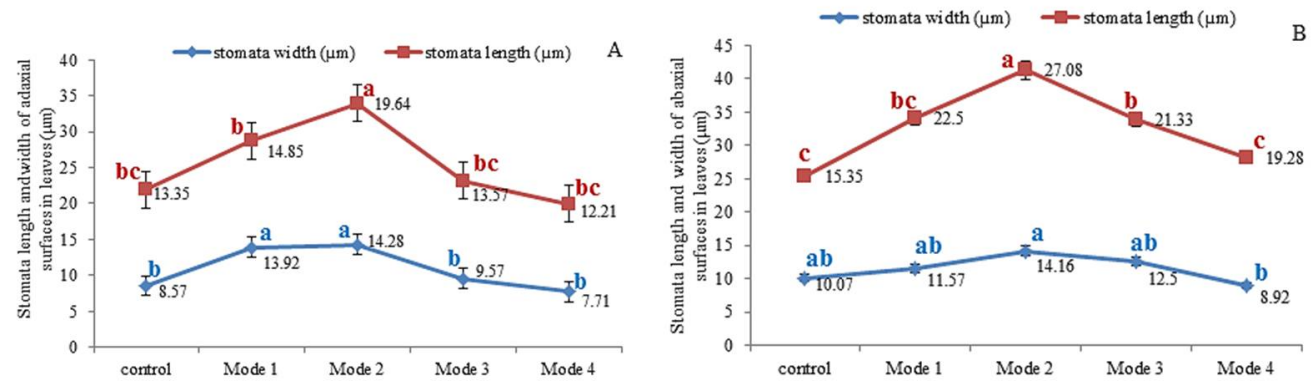

Fig. 5. Stomata sizes (length and width) of adaxial (A) and abaxial (B) surfaces of leaves of tomato plants treated with different light.

The changes in the stomatal activities of a plant that was under different factors showed that they were important as indicators for determining application factors. Carbon dioxide and water vapour resistance are related to stomatal index and stomata sizes (width/length) and it is known that changes in stomatal parameters affect the amount of water and gas exchange in the leaves, and stomatal responses may effects the photosynthesis (Yamori et al. 2016). So, The authors think that the increase or decrease in the leaf parameters with the different light applications can affect $\mathrm{CO}_{2}$ assimilation and evaporation in a positive or negative way. For this reason, the changes of the stomatal responses under light (blue light) changes and being directly proportional to the $\mathrm{CO}_{2}$ reception, showed a close relationship with the growth of the plant (Ishishita et al. 2016).

Chlorophyll pigments which enable the conversion of light energy to stored chemical energy, are also called as the photosynthetic pigment determining amount of solar radiation absorbed by the leaf, and is a very important molecule that ensures the growth, development and differentiation of the plant. They are one of the main components of the photosynthesis mechanism (Stewart and 
Globig 2016). Therefore, the positive relationship between the physiological status of the plant and the amount of chlorophyll (Kosugi et al. 2003) shows that the amount of chlorophyll is the main factor to determine the photosynthethic capacity (Meier et al. 2011). Total chlorophyll content of the tomato leaves at different light applications were observed with monthly measurements (Fig. 6).

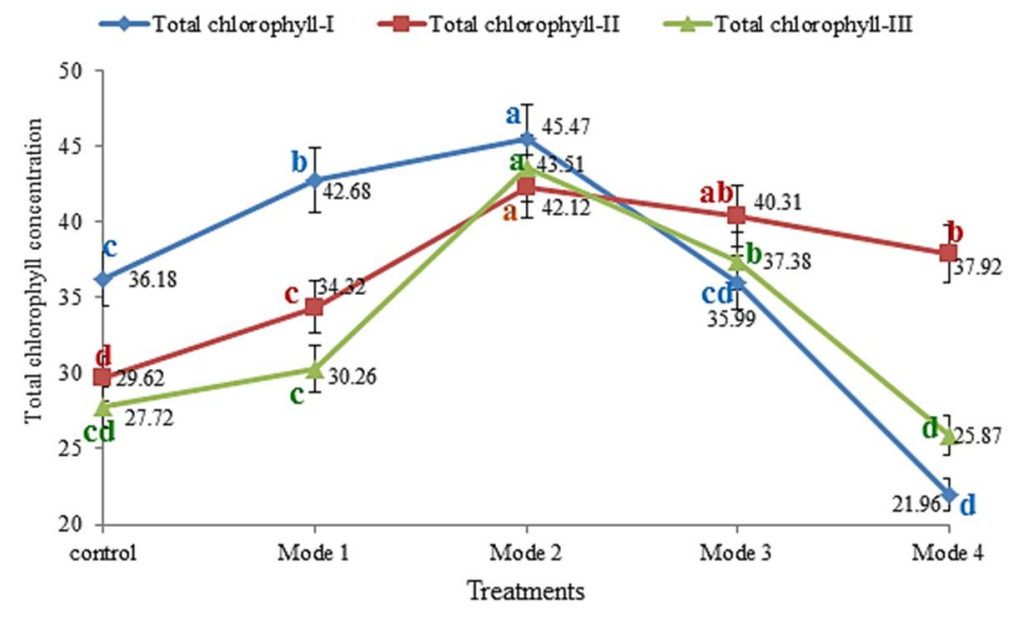

Fig. 6. Total chlorophyll content in leaves of tomato plants treated with different light.

Accordingly as a result of these three measurements it specified that Mode 2 application has given better results than the control and other applications $(\mathrm{p}<0.05)$. As a result of all three measurements, other applications, except Mode 4, gave better results than the control and in Mode 4 application it is determined that tomato plants have lower chlorophyll content than the control.

The results obtained in all the examined parameters were statistically evaluated and the comparisons were performed according to these results. When all the parameters were evaluated it is specified that Mode 2 application is the application which the best results gave in comparison the control application and other applications. The positive correlation between the amount of chlorophyll and the stomatal responses showed that these parameters indicate the physiological properties of the plants. In this work, the morphological, anatomical, physiological responses, the positive relationship between the stomatal responses and chlorophyll amount have been determined. The summation of the different applications based on the stomatal movements and the changes in the amount of chlorophyl of the leaves of the different tomatoes, the order can be formed as Mod2, Mod1, Mod3, the control and Mod4.

\section{Acknowledgements}

The authors wish to express thanks to the authorities of TUBITAK-TEYDEB (2130035) for financial support.

\section{References}

Assmann SM and Jegla T 2016. Guard cell sensory systems: recent insights on stomatal responses to light, abscisic acid, and $\mathrm{CO}_{2}$. Curr. Opinion in Plant Biol. 33: 157-167.

Caponetto R Fortuna L Nunnari G and Occhipinti L 1998. A fuzzy approach to greenhouse climate control.

Proceedings of the American Control Conf. Philadelphia Pennsylvania 1866-1870. 
Esmaeilpour A Labeke M C V Samson R Boeckx P and Damme P V2016. Variation in biochemical characteristics, water status, stomata features, leaf carbon isotope composition and its relationship to water use efficiency in pistachio (Pistacia vera L.) cultivars under drought stress condition. Scientia Horticult. 211: 158-166.

Ishishita K, Suetsugu N Hirose Y Higa T.Doi M Wada M Matsushita T and Gotoh E 2016. Functional characterization of blue-light-induced responses and PHOTOTROPIN 1 gene in Welwitschia mirabilis. J. Plant Res. 129: 175-187.

Kosugi Y Shibata S and Kobashi S 2003. Parameterization of the $\mathrm{CO}_{2}$ and $\mathrm{H}_{2} \mathrm{O}$ gas exchange of several temperate deciduous broad- leaved trees at the leaf scale considering seasonal changes. Plant Cell Environ. 26: 285-301.

Meier S Tzfadia O Vallabhaneni R Gehring C and Wurtzel E T 2011. A transcriptional analysis of carotenoid, chlorophyll and plastidial isoprenoid biosynthesis genes during development and osmotic stress responses in Arabidopsis thaliana. BMC Systems Biol. 5: 1-19.

Nhut DT, Nam NB 2010. Light-Emitting Diodes (LEDs): An Artificial Lighting Source for Biological Studies. In: Proceedings of the 3rd international conference of the development of BME in Vietnam. 11-14 January 2010.

Rengifo E Urich R and Herrera A 2002. Water relations and leaf anatomy of the tropical species, Jatropha gossypifolia and Alternanthera crucis, grown under elevated $\mathrm{CO}_{2}$ concentration. Photosynthetica 40: 397-403.

Sack L and Scoffoni C 2013. Leaf venation: structure, function, development, evolution, ecology and applications in the past, present and future. New Phytol. 198: 983-1000.

Salgado P and Cunha J B 2005. Greenhouse climate hierarchical fuzzy modelling. Control Engineering Practice 13: 613-628.

Stewart P and Globig S 2016. Photosynthesis: research progress in botany; in genetic, environmental and evolutionary aspects. Apple Academic Press. Inc., 3333 Canada.

Taiz L and Zeiger E 2010. Plant Physiology. 5th Ed. Sinauer Associates, Sunderland.

Wit M Galvão V C and Fankhauser C 2016. Light-mediated hormonal regulation of plant growth and development. Plant Growth Develop. 67: 513-537.

Yamori W Makino A and Shikanai T 2016. A physiological role of cyclic electron transport around photosystem I in sustaining photosynthesis under fluctuating light in rice. Scie. Rep. 6: 20147.

Zhao W Sun Y Kjelgren R and Liu X 2015. Response of stomatal density and bound gas exchange in leaves of maize to soil water deficit. Acta Physiol. Plant. 37: 1704.

Zhou Z Xiangya T and Shuhe Z 2016. Light regulation system for greenhouse based on light requirements of crops. J. Agricul. Mechanization Res. 2016, 02.

(Manuscript received on 27 February, 2018; revised on 7 October, 2020) 